OPEN ACCESS

Edited by:

Francesca Taraballi, Houston Methodist Research Institute,

United States

Reviewed by:

Michel M. Maharbiz, University of California, Berkeley,

United States

Rajendra K. Singh,

Institute of Tissue Regeneration

Engineering (ITREN), South Korea

${ }^{*}$ Correspondence:

Fabio Benfenati

fabio.benfenati@it.it

tThese authors have contributed equally to this work

$¥$ Present address:

Bruno F. E. Matarèse, Department of Chemical Engineering and Biotechnology, West Cambridge

Site, Cambridge, United Kingdom Paul L. C. Feyen,

Department of Biology, Neuroscience Institute, University of Texas at San Antonio, San Antonio, TX United States

Specialty section: This article was submitted to Biomaterials,

a section of the journal Frontiers in Bioengineering and Biotechnology

Received: 07 July 2019 Accepted: 03 October 2019 Published: 22 October 2019

Citation:

Matarèse BFE, Feyen PLC, de Mello JC and Benfenati F (2019) Sub-millisecond Control of Neuronal Firing by Organic Light-Emitting

Diodes.

Front. Bioeng. Biotechnol. 7:278. doi: 10.3389/fbioe.2019.00278

\section{Sub-millisecond Control of Neuronal Firing by Organic Light-Emitting Diodes}

\author{
Bruno F. E. Matarèse ${ }^{1 \neq \neq}$, Paul L. C. Feyen ${ }^{2,3 \neq \neq}$, John C. de Mello ${ }^{1,4}$ and Fabio Benfenati ${ }^{2,5 *}$ \\ ${ }^{1}$ Department of Chemistry, Imperial College London, South Kensington Campus, London, United Kingdom, ${ }^{2}$ Center for \\ Synaptic Neuroscience and Technology, Istituto Italiano di Tecnologia, Genoa, Italy, ${ }^{3}$ Section of Physiology, Department of \\ Experimental Medicine, University of Genova, Genoa, Italy, ${ }^{4}$ Centre for Organic Electronic Materials, Department of \\ Chemistry, Trondheim, Norway, ${ }^{5}$ IRCCS Ospedale Policlinico San Martino, Genoa, Italy
}

Optogenetics combines optics and genetics to enable minimally invasive cell-type-specific stimulation in living tissue. For the purposes of bio-implantation, there is a need to develop soft, flexible, transparent and highly biocompatible light sources. Organic semiconducting materials have key advantages over their inorganic counterparts, including low Young's moduli, high strain resistances, and wide color tunability. However, until now it has been unclear whether organic light emitting diodes (OLEDs) are capable of providing sufficient optical power for successful neuronal stimulation, while still remaining within a biologically acceptable temperature range. Here we investigate the use of blue polyfluorene- and orange poly(p-phenylenevinylene)-based OLEDs as stimuli for blue-light-activated Sustained Step Function Opsin (SFFO) and red-light-activated ChrimsonR opsin, respectively. We show that, when biased using high frequency (multi-kHz) drive schemes, the OLEDs permit safe and controlled photostimulation of opsin-expressing neurons and were able to control neuronal firing with high temporal-resolution at operating temperatures lower than previously demonstrated.

\footnotetext{
Keywords: organic light-emitting diodes, optogenetics, neurons, pulsed-operation, optical stimulation, bioelectronics, photoexcitation, electrophysiology
}

\section{HIGHLIGHTS}

- Successful activation of Sustained Step Function Opsin (SFFO) by blue polyfluorene-OLEDs.

- Successful activation of ChrimsonR opsin by orange poly(p-phenylenevinylene)-OLEDs.

- Pulsed-mode operation allows neuronal stimulation in a compatible temperature range.

- Safe and controlled millisecond control of firing in opsin-expressing neurons by OLEDs.

\section{INTRODUCTION}

Optogenetics involves the genetic modification of a cell to enable optical control of ion currents across the plasma membrane by light-gated ion channels or transporters. The applications of optogenetics are diverse and include the ability to investigate neural circuits with high spatiotemporal control, the treatment of a wide range of degenerative and paroxysmal pathologies, the regulation of artificial organ function, or the triggering of bio-hybrid robot movements 

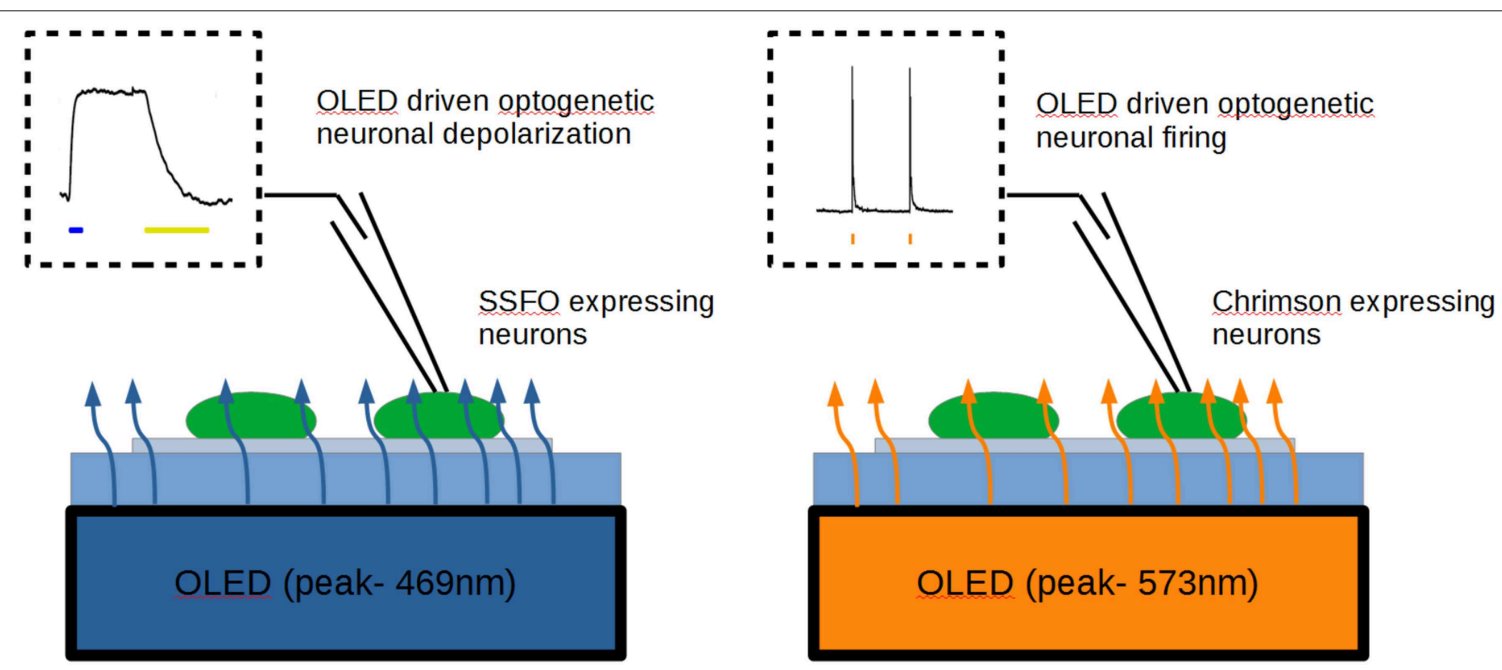

GRAPHICAL ABSTRACT | Using blue polyfluorene- (left) and orange poly(p-phenylenevinylene) (right) OLEDs in pulsed-mode operation, we obtained a successful activation of Sustained Step Function Opsin (SFFO) and ChrimsonR opsin, respectively, yielding depolarization and millisecond control of firing in opsin-expressing neurons by OLEDs.

(Toettcher et al., 2011; Reinbothe et al., 2014; CehajicKapetanovic et al., 2015; Paz and Huguenard, 2015; Park et al., 2016). The power of optogenetics derives in part from its ability to genetically target specific cell types and also from the availability of a broad range of protein tools that have been engineered to tune kinetics, ion selectivity, optical response, and wavelength sensitivity (Mattis et al., 2011; Yizhar et al., 2011; Klapoetke et al., 2014; Gaub et al., 2015). With the technology already being used in a clinical testing for the treatment of degenerative blindness (see RST-001 Phase I/II Trial for Retinitis Pigmentosa, 2016), there is a recognized need to develop thin, flexible, efficient, multi-color biocompatible light sources for both wearable and implantable devices (Park et al., 2009; Someya et al., 2016).

Spectrally matched stimulation of photosensitive proteins is typically carried out with filtered halogen lamps, lasers or inorganic light emitting diodes, with the light beam commonly delivered via microscopes or by coupling to optical fibers (Dal Maschio et al., 2010; Welkenhuysen et al., 2016). There have also been several reports involving the use of arrays of micro LEDs to target individual neurons with high spatial precision (Grossman et al., 2010; Gossler et al., 2014; Soltan et al., 2014; Qazi et al., 2018). While the initial results were promising, various drawbacks including the bulky and rigid features of inorganic LED sources, heat production, decrease in efficiency or mismatched spectral characteristics (Krames et al., 2007; Tsintzos et al., 2008; Fan and Li, 2015) have so far prevented the development of fully implantable devices.

Abbreviations: CN-PPV, poly(2,5-di(hexyloxy)cyanoterephthalylidene); DI, double distilled; DIV, days in vitro; HEK293T, Human Embryonic Kidney293T; EPD50, effective power density for 50\% activation; IPA, isopropanol; ITO, indium tin oxide; MEH-PPV, poly[2-methoxy-5-(2-ethylhexyloxy)1,4-phenylenevinylene]; OLED, organic light-emitting diodes; PEDOT:PSS, poly(3,4-ethylendioxythiophene:poly(styrene-sulfonate); PFO, poly(9,9-din-octylfluorenyl-2,7-diyl); PVK, poly(9-vinylcarbazole); SO-PPV, phenyl alkoxyphenyl-based PPV copolymer Livilux PDO-124; SSFO, sustained step function opsin.
By contrast, organic light-emitting diodes (OLEDs) can be fabricated using layers of soft conformable materials that are well-matched to the prerequisites of implantable devices. The thin layers used in the OLED device stack add minimal (submicron) thickness to the chosen substrate, making it feasible to incorporate OLEDs in many locations where larger lightsources could not be tolerated (Yang et al., 2012; Zhang et al., 2015). Moreover OLEDs can be processed onto a wide range of substrate materials, allowing for the fabrication of flexible (Yokota et al., 2016) and stretchable devices (Someya et al., 2016; Yin et al., 2016). Using standard processing methods, it is possible to fabricate homogeneously emitting, pixelated surfaces covering microscopic $\left(\mu \mathrm{m}^{2}\right)$ to macroscopic $\left(\mathrm{cm}^{2}\right)$ length scales (Sandström et al., 2012; Zheng et al., 2013), with overall device thicknesses as low as $2 \mu \mathrm{m}$ (White et al., 2013). From a biological perspective, this favorably positions the technology for many useful tasks, ranging from highly localized sub-cellular targeting to organ targeting (Toettcher et al., 2011; Bruegmann et al., 2015). Rapid progress has been made in applying organic electronics to optogenetic activation in systems including green algae, Drosophila larvae and Human Embryonic Kidney-293T (HEK293T) cells (Steude et al., 2015, 2016; Morton et al., 2016) but, until now, the chosen optical stimuli have lasted seconds to minutes, limiting the range of optogenetic applications (Smith et al., 2014; Blain Christen et al., 2016). The performances of OLEDs are progressing rapidly and have now reached the market enabling new display and lighting application (e.g., smartphone display and large-size OLED TVs), but their operating optical power and temporal resolution for optogenetics requirements are insufficiently investigated. In particular, the feasibility of using sub-millisecond frequency biasing schemes to improve electroluminescence efficiencies, and hence permit short-timescale stimulation of opsins, has not previously been investigated.

One key challenge toward the long-term goal of developing an implantable OLED-based optical stimulus for controlling neural 
activity is to identify simple and biocompatible OLED structures that are capable of eliciting biological effects. Here, we report, for the first time, the use of poly(p-phenylene vinylene)-based and polyfluorene-based light emitting polymers for generating polymeric OLEDs for opsin stimulation. New possibilities for organ targeting stimulation are offered by homogeneous solution-processed large-area polymeric OLED pixels that are difficult to achieve by non-solution processed OLEDs or their inorganic LED counterparts. Moreover, high elastic modulus and biological inertness of carbon-based polymers confer major advantages for use in non-conformal body cavities.

Using extended multi-kHz pulse trains, we demonstrate an effective control of the firing rate of primary neurons expressing microbial opsins in vitro on a millisecond time scale.

To test our OLEDs, two opsins were selected from the huge toolbox of opsin variants characterized by distinct spectral sensitivity and different kinetics, namely the blue-sensitive, bistable Sustained Step-Function Opsin (SSFO; Yizhar et al., 2011) to induce multi-second neuronal depolarization with high sensitivity, and the red-sensitive, fast kinetics ChrimsonR (Klapoetke et al., 2014) to induce a sub-millisecond control of neuronal firing. The results reported here confirm the feasibility of using OLEDs operating at low, biologically compatible temperatures to control neural activity.

\section{MATERIALS AND METHODS}

\section{Materials}

Materials employed: MEH-PPV (poly[2-methoxy-5-(2ethylhexyloxy)-1,4-phenylenevinylene]) for F(4); CN-PPV [poly(2,5-di(hexyloxy)cyanoterephthalylidene)] for $\mathrm{F}(5)$; $\mathrm{PFO}$ [poly(9,9-di-n-octylfluorenyl-2,7-diyl)]; and PVK [poly(9vinylcarbazole)] for $F(1)$ were ordered from Sigma Aldrich. Super Orange (SO): Phenyl alkoxyphenyl PPV copolymer, conjugated polymers Livilux ${ }^{\mathrm{TM}}$ PDO-124 (SO-PPV) for $\mathrm{F}(2$ ) devices were obtained from Merck PPF. For biocompatibility assays, thin films of fluorescent polymers (PFO, PVK, SOPPV) were prepared by dissolving $6 \mathrm{mg}$ material in $1 \mathrm{~mL}$ Chlorobenzene. Glass coverslips were spin-coated for $30 \mathrm{~s}$ at $1,200 \mathrm{RPM}$, and annealed at $150^{\circ} \mathrm{C}$ for $20 \mathrm{~min}$.

\section{OLED Fabrication}

Conventional solution processing organic light emitting diodes were fabricated on glass by coating indium tin oxide (ITO; $10 \mathrm{ohm} / \mathrm{sq}$ ) with a $30 \mathrm{~nm}$ layer of $\operatorname{poly}(3,4-$ ethylendioxythiophene:poly(styrenesulfonate) (PEDOT:PSS). All spin coating steps were carried out in air. Cleaning of the ITO slides; film coating and electrode deposition were carried out all the same day to ensure optimum results. ITO coated glass substrates were supplied by Xin Yan Technology Ltd. The glasses were sonicated in standard acetone and then in soap and doubledistilled (DI) water for $20 \mathrm{~min}$, in DI water 3 times for $5 \mathrm{~min}$, in acetone twice for $5 \mathrm{~min}$ and in isopropanol (IPA) for $5 \mathrm{~min}$, followed by blow-drying of the substrates. The ITO substrates were treated by oxygen plasma treatment. The PEDOT:PSS layers were deposited by spin coating at a spin speed of 3,500 rpm for $40 \mathrm{~s}$ and then annealed at $150^{\circ} \mathrm{C}$ for $20 \mathrm{~min}$. Polymeric devices followed conventional PLED structure using PEDOT:PSS for the injection of holes from the ITO anode and Calcium for the injection of electron from the Aluminum cathode. The precursor solutions were prepared the day before device fabrication and the solutions were stirred at $<50^{\circ} \mathrm{C}$ overnight. The precursor solutions of fluorescent polymers (PFO, PVK, SO-PPV, MEH$\mathrm{PPV}$, and $\mathrm{CN}-\mathrm{PPV}$ ) were prepared by dissolving $6 \mathrm{mg}$ material in $1 \mathrm{~mL}$ chlorobenzene and were spin-coated for $30 \mathrm{~s}$ at 1,200 RPM on top of PEDOT:PSS. Following active layers deposition, Calcium $(25 \mathrm{~nm})$ and aluminum $(100 \mathrm{~nm})$ top-electrodes were deposited onto all the devices by thermal evaporation through a shadow mask at 10-6 mbar. Active areas measured $75 \mathrm{~mm}^{2}$. The encapsulation of the devices was effected manually using another glass substrate of $1.1 \mathrm{~mm}$ adhered onto the device using flexible sealants from DELO-KATIOBOND and a low power UV light to set the glue.

\section{OLED Characterization}

The electrical characteristics of the devices encapsulated were measured in air at room temperature using a Keithley 2450 electrometer for the electrical current-voltage measurement. Emission spectra were measured using a fiber-coupled Ocean Optics $2000+$ spectrometer. Absolute optical power intensity was determined using a large area calibrated Si PIN Photodiode (28 $\times 28 \mathrm{~mm}$ active area, from Hamamatsu) coupled to a Keithley 6517A electrometer. An OPT101P Photodetector Amplifier from Texas Instruments connected to the National Instruments NI PCI-5112 100MHz Digital Oscilloscope Board was used to detect fast pulsing mode imposed by the Arduino Uno microcontroller. Surface temperature was measured and digitized by placing a thermistor (TC-3444B, Warner Instruments) in contact with the OLED anode-side glass surface. Output voltage trace responses were analyzed in Matlab (Mathworks). For recordings in culture medium, $500 \mu \mathrm{l}$ of Hank's Balanced Salt Solution (HBSS) was used to immerse the sensor.

\section{Primary Neuronal Cultures}

Polymeric films and glass culture substrates were thermally sterilized at $120^{\circ} \mathrm{C}$, prior to overnight incubation in $0.1 \%$ polyL-lysine solution. Primary cultures of hippocampal neurons were prepared from embryonic 18-day rat and mouse embryos (Charles River). Briefly, hippocampi or cortex were dissociated by a 15 -min incubation with $0.25 \%$ trypsin at $37^{\circ} \mathrm{C}$ and cells were plated on poly-L-lysine-coated substrates $(0.1 \%$ PLL in Borax solution overnight) in Neurobasal supplemented with $2 \mathrm{mM}$ L-glutamine, 2\% B27, $100 \mu \mathrm{g} / \mathrm{ml}$ penicillin and $100 \mu \mathrm{g} / \mathrm{ml}$ streptomycin, and with $10 \%$ horse serum (Life Technologies) in the first $4 \mathrm{~h}$ of plating. Cultures were maintained at $37^{\circ} \mathrm{C}$ in a humidified atmosphere containing $5 \% \mathrm{CO}_{2}$.

\section{Expression of Opsins in Neurons}

Neurons were transfected by Lipofectamine 2000 (Thermo Fischer Scientific) reagent using a $1 \mathrm{~h}$ incubation time and supplier protocol. pAAV-Ef1a-DIO hChR2(C128S/D156A)EYFP was a gift from Karl Deisseroth (Addgene plasmid \# 35503). FCK-ChrimsonR-GFP was a gift from Edward Boyden (Addgene plasmid \# 59049). Third-generation lentiviruses 
were produced by transient four-plasmid co-transfection into HEK293T cells using the calcium phosphate transfection method. Supernatants were collected, passed through a $0.45 \mu \mathrm{m}$ filter and purified by ultracentrifugation.

\section{Electrophysiology and Light Stimulation Protocols}

Whole-cell patch-clamp recordings of cultured neurons were performed at room temperature using patch pipettes (4-8 $M \Omega$ ), after attaining $G \Omega$ patch seals. Traces were acquired in current-clamp mode using HEKA EPC10 amplifier and digitizer, and Patchmaster software (HEKA). The extracellular solution contained $\mathrm{NaCl}(135 \mathrm{mM}), \mathrm{KCl}(5.4 \mathrm{mM}), \mathrm{MgCl}_{2}(1 \mathrm{mM}), \mathrm{CaCl}_{2}$ $(1.8 \mathrm{mM})$, HEPES $(5 \mathrm{mM})$, and glucose $(10 \mathrm{mM})$, and was adjusted to $\mathrm{pH} 7.4$ with $\mathrm{NaOH}$. The intracellular solution contained K-gluconate (126 mM), $\mathrm{KCl}(4 \mathrm{mM}), \mathrm{MgSO}_{4}(1 \mathrm{mM})$, $\mathrm{CaCl}_{2}(0.02 \mathrm{mM})$, BAPTA $(0.1 \mathrm{mM})$, glucose $(15 \mathrm{mM})$, HEPES $(5 \mathrm{mM})$, ATP $(3 \mathrm{mM})$ and GTP $(0.1 \mathrm{mM})$, and was adjusted to $\mathrm{pH} 7.3$ with $\mathrm{KOH}$. Responses were amplified, low-pass-filtered at $3.9 \mathrm{kHz}$, digitized at $20-50 \mathrm{kHz}$, stored, and analyzed with Matlab (Mathworks). For photoactivation of young neurons expressing SSFO, $500 \mathrm{~ms}$ pulses were applied with either full duty cycle continuous DC bias, or by $50 \%$ duty cycle stimulus with sub pulse frequencies of $10 \mathrm{kHz}, 5 \mathrm{kHz}, 50 \mathrm{~Hz}$. For experiments on mature neurons expressing SSFO, 2,000 ms pulses driven with $10 \mathrm{kHz}$ sub-pulses at a $50 \%$ duty cycle were employed. Experiments on SO-PPV based device for excitation of ChrimsonR-expressing culture employed $50 \mathrm{~ms}$ and $2,000 \mathrm{~ms}$ stimuli, composed of $10 \mathrm{kHz}$ sub-pulses at $50 \%$ duty cycle. Unless otherwise stated, a $30 \mathrm{~V}$ bias was employed to drive the organic semiconductor based diodes.

\section{Safety}

No unexpected significant hazards or risks were associated with the reported work. All animal manipulations and procedures were performed in accordance with the guidelines established by the European Community Council (Directive 2012/63/EU of 22 September 2010) and were approved by the Italian Ministry of Health.

\section{Statistical Analysis}

Statistical tests were selected based on the data distribution. Normal distribution of the data was verified using the D'Agostino and Pearson omnibus normality test $(p$-value $=0.05)$. The sample size was indicated as number of recorded neurons (n). Analysis was carried out with Graphpad Prism 5.01 (Graphpad Software Inc.).

\section{RESULTS}

\section{Fabrication and Biocompatibility of OLEDs Used for Optogenetic Activation}

Two optogenetic actuators were investigated for neural control: the blue light-activated, bi-stable SSFO (Yizhar et al., 2011) and the red light-activated ChrimsonR (Klapoetke et al., 2014). Both actuators are light-gated cation channels targeted to the cell membrane, whose activation results in inward (depolarizing) currents when expressed in neuronal cells. Following a screening of candidate light-emitters capable of matching the activation spectra of the two opsins (taking into account emission spectra, achievable emission intensities, and commercial availability of the materials, Figure S1), the blue fluorene-based polymer poly(9,9-di-n-octylfluorenyl-2,7-diyl) (PFO) was selected for SSFO activation, and the orange phenyl alkoxyphenyl based PPV copolymer Livilux PDO-124 (SO-PPV) was selected for ChrimsonR activation (Figures 1A,B). Blue OLEDs were fabricated by sequentially spin-casting onto indium tin oxide (ITO)-coated glass layers of poly(3,4-ethylendioxythiophene : poly(styrene-sulfonate) (PEDOT:PSS) as an anode modifier, poly(9-vinylcarbazole) (PVK) as a hole-transporter and electronblocker, and PFO as a blue emissive layer (peak emission: $469 \mathrm{~nm}$ ); orange OLEDs were fabricated by sequentially spincasting layers of PEDOT:PSS and SO-PPV as an orange emissive layer (peak emission: $573 \mathrm{~nm}$ ). Both sets of devices were completed by evaporating an aluminum-capped calcium cathode on top of the emissive layer, prior to encapsulation of the exposed surface using UV-curable epoxy resin and a glass cover-slide. The devices were rendered translucent in the near infrared range by restricting the thickness of the $\mathrm{Al}$ capping layer to $<100 \mathrm{~nm}$, resulting in a full device transmission of $\sim 5 \%$ for the blue OLED and $\sim 4 \%$ for the orange OLED at $650 \mathrm{~nm}$ and $\sim 8 \%$ for the blue OLED and $\sim 7 \%$ for the orange OLED at $850 \mathrm{~nm}$ (peak). In this way, it was possible to directly image neurons and carry out patchelectrode measurements using a standard patch-clamp set-up equipped with an infrared transmission light path coupled to a CCD camera (Figures 1C-E).

The main challenge is to ensure that OLEDs can operate in a highly saline and biologically active aqueous environment. Finding materials that are inherently stable in the environment in which they function is key to device optimization. We recently demonstrated the photophysical stability of light emitting polymers after extensive immersion to cell-culture medium (Matarèse, 2017). In addition, we have demonstrated the stability and biocompatibility of key OLED electrodes (ITO, gold, aluminum, and silver) when exposed to the physiological milieu (Falco et al., 2016; Matarèse et al., 2018). Nonetheless, the difficult challenge of implantable fully biocompatible OLEDs remain on the electrode injection materials used in devices that are inherently very sensitive to moisture and oxygen (Matarèse, 2017).

In the devices used for this study, the active materials are isolated from the biological medium by a thin glass encapsulant. While the long-term goal is to achieve fully encapsulated and biocompatible devices for implantation, we aimed at demonstrating the suitability of OLEDs for neuronal stimulation to yield the necessary characterization of the encapsulated device for its ability to induce a reliable and high temporalresolution control of neuronal activity in vitro. In view of future developments of flexible devices for bio-implantation, we also preliminarily assessed the cell biocompatibility of the active emissive layer materials that may occasionally get in contact with the biological environment in vivo.

When the viability and the basic physiological properties of primary hippocampal neurons on spin-coated polymeric films were measured, no significant differences between neurons 
A

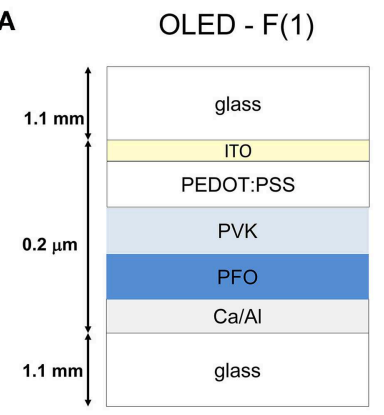

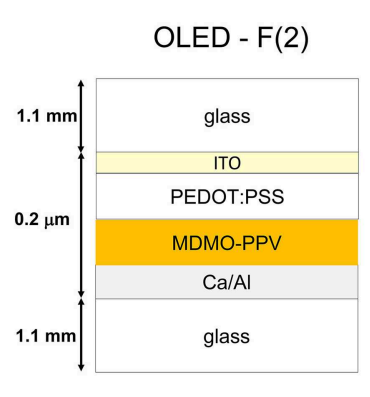

B
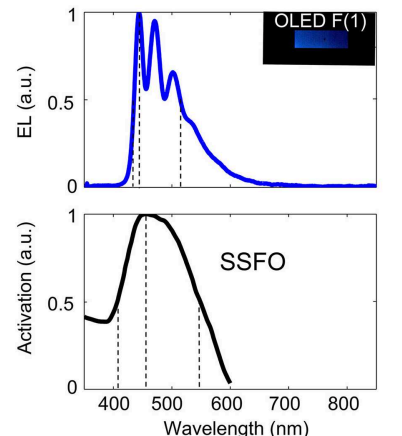
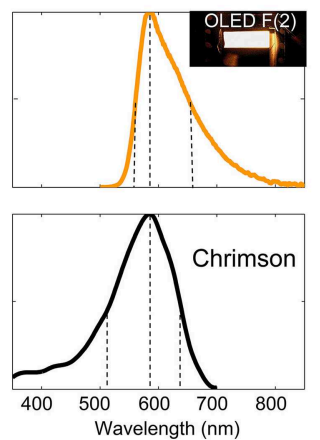

C

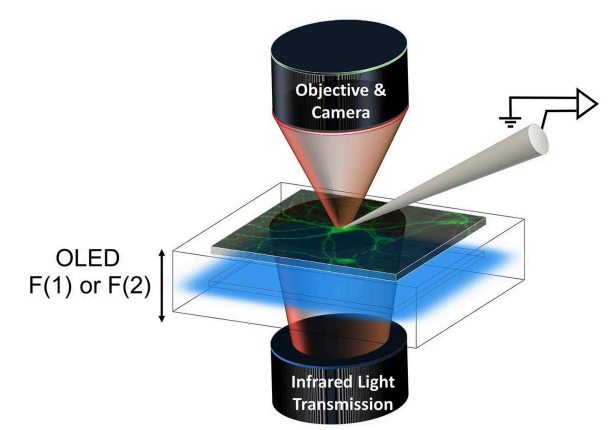

D

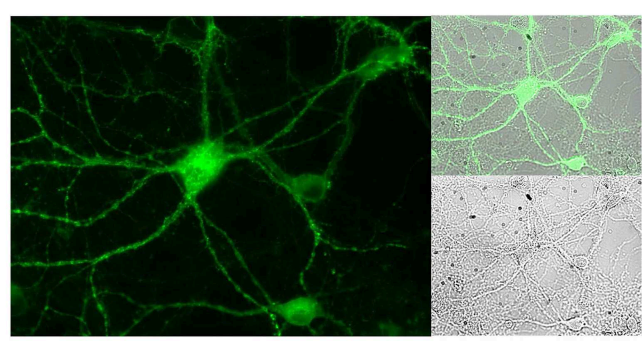

E

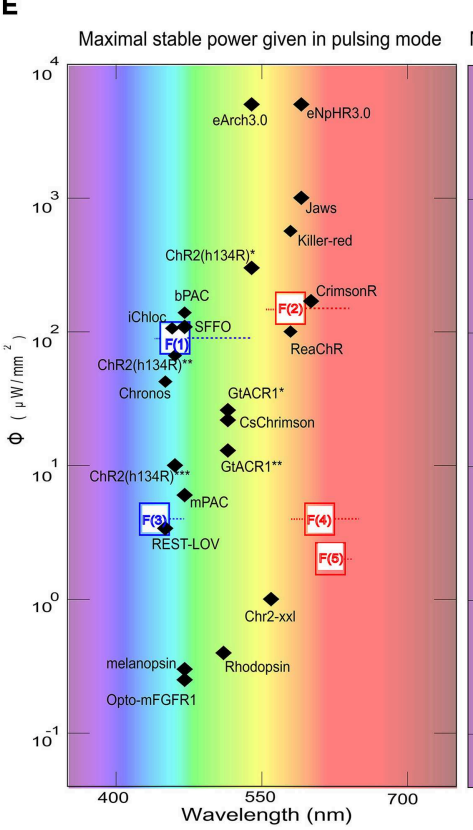

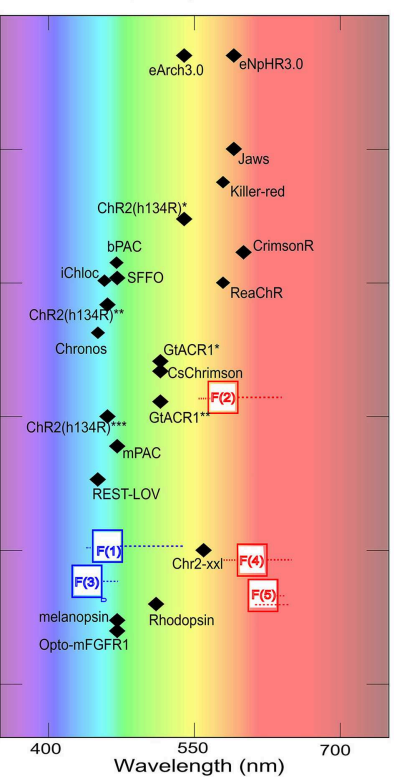

FIGURE 1 | Blue and orange OLEDs based on PFO and SO-PPV use for photo-activation of SSFO and ChrimsonR, respectively. Device architectures (A) and emission spectra (B) for blue PFO and orange SO-PPV OLEDs. Due to the use of a thin ( $<100 \mathrm{~nm})$ Al-capping layer, both sets of devices were translucent in the near infrared range, allowing simultaneous photo-stimulation and patch-clamp recordings guided by infrared transmission and epifluorescence. (C) Due to the use of a thin $(<100 \mathrm{~nm})$ Al-capping layer, both sets of devices were translucent in the near infrared range, allowing simultaneous photo-stimulation and patch-clamp recordings guided by infrared transmission and epifluorescence. (D) Fluorescence images of neurons expressing eGFP and located at the surface of the blue PFO OLED, alongside the near infrared transmission image. (E) Maximal optical output of solution-processed blue and red-shifted OLEDs with respect to the activation requirements of a toolbox of optogenetic probes. Fluorescent OLEDs are represented with their active layer number $\mathrm{F}(\mathrm{x})$ and following Glass/TO/Pedot:Pss/F(x)/Ca/Al structure with PFO and PVK for F(1) devices; Super Orange (SO-PPV) for F(2) devices; PFO for F(3); MEH-PPV for F(4); CN-PPV for F(5). References for OPSIN tools are: ChR2-XXL (Dawydow et al., 2014); Rhodopsin (Cehajic-Kapetanovic et al., 2015); Jaws (Chuong et al., 2014); ChR2(H134R) (Yu et al., 2015); Killer Red (Williams et al., 2013); GtACR1 ${ }^{\star}$, GtACR1 $^{* \star}$ (Govorunova et al., 2015); CsChrimson (Mohammad et al., 2017); Melanopsin (Beiert et al., 2014); bPAC (Stierl et al., 2011); REST-LOV (Paonessa et al., 2016); ChR2(H134R)* (Reinbothe et al., 2014); C1V1(E162T) (Packer et al., 2012); mPAC (Raffelberg et al., 2013); Opto-mFGFR1 (Grusch et al., 2014); ChR2(H134R)** (Maimon et al., 2017); ReaChR1 (Kaufmann et al., 2017); eNpHR3.0, eArch3.0 (Mattis et al., 2011); Chronos (Klapoetke et al., 2014); iChloC (Wietek et al., 2015).

grown on the polymers and those grown on glass controls were observed (Figure S2), confirming the biocompatibility of the used materials, similarly to what previously shown for other organic semiconductors (Perylene P13, P3HT; Ghezzi et al., 2011, 2013; Toffanin et al., 2013).

\section{Performance of OLEDs Under Pulsed Excitation}

Under typical DC operating biases of $<8 \mathrm{~V}$, OLEDs commonly provide optical output power densities ranging from hundreds to tens of thousands of $\mathrm{nW} / \mathrm{mm}^{2}$ (Lochner et al., 2014; Smith et al., 2014; Morton et al., 2016). However, the typical excitation levels required for optogenetics range from hundreds to thousands of $\mu \mathrm{W} / \mathrm{mm}^{2}$ (Mattis et al., 2011; Dawydow et al., 2014; Reinbothe et al., 2014; Mohammad et al., 2017). For instance, under continuous bias operation, our blue OLEDs emitted a peak optical power of $15 \mu \mathrm{W} / \mathrm{mm}^{2}$ at $8 \mathrm{~V}$, while our orange OLEDs emitted a peak optical power of $35 \mu \mathrm{W} / \mathrm{mm}^{2}$ at $15 \mathrm{~V}$ (Figure 2A). Increasing the DC bias further resulted in rapid degradation of the devices due to excessive Joule heating, and 


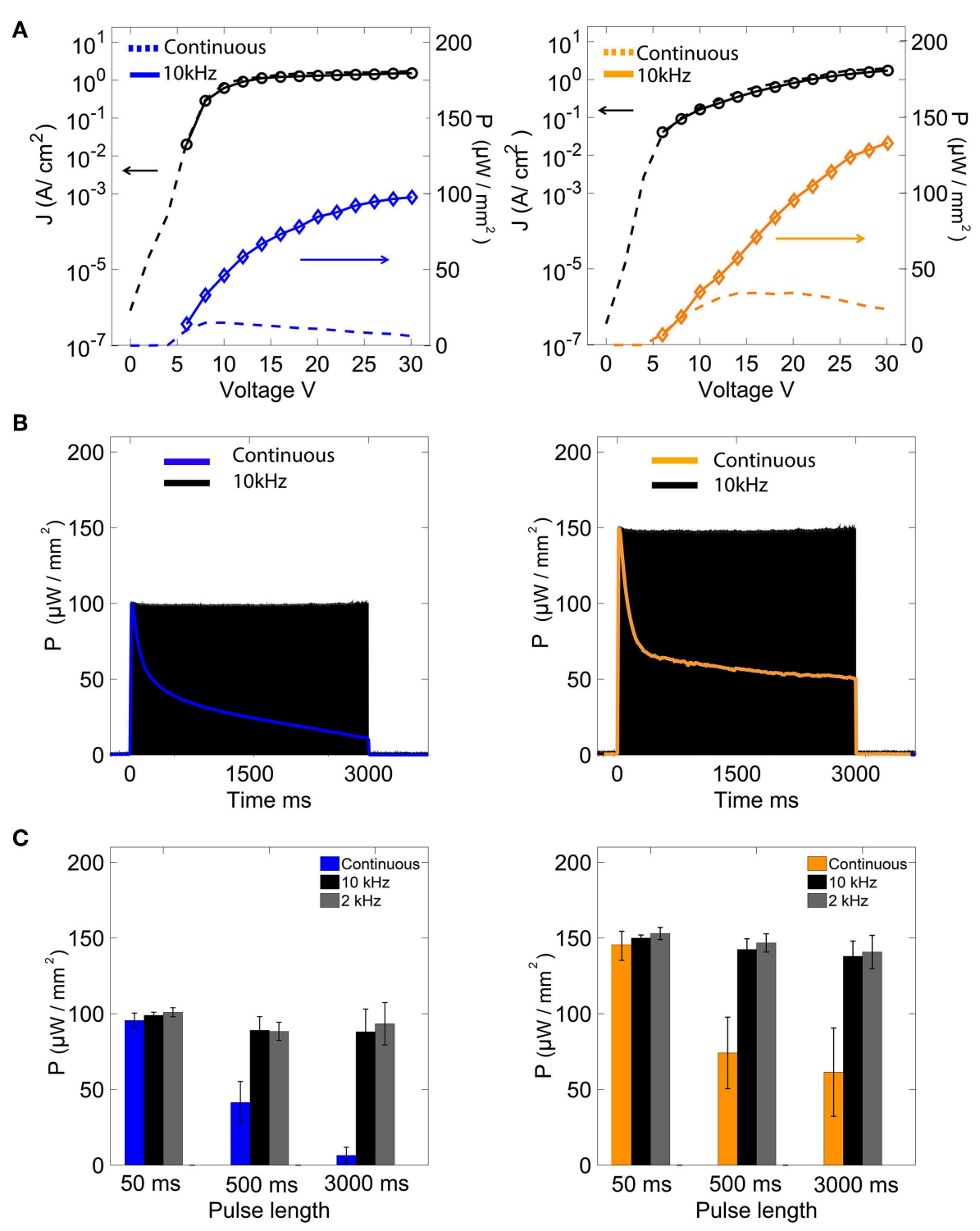

FIGURE 2 | Performance characteristics of blue and orange OLEDs. (A) Current-density and optical power vs. voltage characteristics for blue and orange devices under DC and $10 \mathrm{kHz}$ square-wave operation. (B) Optical output power vs. time for blue and orange OLEDs under DC and $10 \mathrm{KHz}$ square-wave operation. (C) Peak optical power (mean \pm sem) under DC and square-wave operation for measurement times of varying length.

hence higher optical powers could not be reliably achieved under DC conditions.

Pulsed mode operation-in which devices are driven using low to moderate duty cycle high frequency biases-has previously been shown to improve the efficiency of OLEDs, allowing the use of lower drive voltages and/or current densities for a given optical output power (Nakanotani et al., 2005). This was found to be the case for both our blue and orange OLEDs which, compared to DC operation at the same voltage, showed slightly lower current densities and substantially higher output powers when driven with a $10 \mathrm{kHz}$ square wave in the range $6-30 \mathrm{~V}$ (Figure 2A). Pulsed mode operation has the further advantage of improving the operating lifetime of OLEDs with respect to continuous operation: under DC operation at $30 \mathrm{~V}$, the output power of our devices dropped to $50 \%$ of the starting value within $\sim 250 \mathrm{~ms}$; under square-wave operation at the same voltage, by contrast, the output power remained approximately constant for over $3 \mathrm{~s}$ (retaining $\sim 98 \%$ of the starting value; Figure 2B). Moreover, using pulsed operation, devices could still operate upon repetition of the protocol over $30 \mathrm{~min}$. Figure $2 \mathrm{C}$ shows the average of each peak optical power over time of the blue and orange OLEDs operated under DC and square-wave operation (two test frequencies of 2 and $10 \mathrm{kHz}$ ) over operating times of 50,500 , and 3,000 ms. For operating times $<50 \mathrm{~ms}$, the devices were stable in both DC and square-wave modes, with outputs of about 100 and $150 \mu \mathrm{W} / \mathrm{mm}^{2}$ at $30 \mathrm{~V}$ for the blue and orange 
OLEDs, respectively. However, for longer operating times, a clear difference was observed between DC and square-wave operation. For instance, after $3 \mathrm{~s}$ of DC operation, the optical output powers of the blue and orange OLEDs had decreased by approximate factors of ten and two; under $3 \mathrm{~s}$ of square-wave operation, by contrast, the optical powers had dropped by $<0.9$ percent for both OLEDs (Figure 2C).

Low values of external quantum efficiency (EQE) of OLEDs are observed at high current densities $\left(>1 \mathrm{~A} / \mathrm{cm}^{2}\right)$ for all devices under $\mathrm{kHz}$ and $\mathrm{DC}$ conditions (Figure S3). It is clear that operation at $10 \mathrm{kHz}$ allows for much longer operation of the OLED compared to DC conditions, but it does not appear to improve massively the quantum efficiency for short pulse lengths. For both OLED devices, the longer was the illumination pulse, the lower the external quantum efficiency was observed (Figure S3 and Table 1).

\section{Pulsed Operation Reduces the Increase in Surface Temperature}

When used in vivo, Joule heating within the OLEDs could elevate the local temperature to levels that may become potentially harmful to cells and tissues. Moreover, a temperature increase can induce changes in membrane capacitance and conductance that would depolarize neurons and thus confound the effects of OLED-stimulated opsins. The maximum temperature induced by an LED is going to depend not only on the power dissipated in the LED, but also the heat capacity of the apparatus and how it is in thermal contact with its surroundings. Here, we only focused on surface temperature as control measurement of sub-millisecond single pulse for this organic LED-optogenetics platform.

We monitored temperature as control measurement for the following in-vitro experiments using a thermocouple positioned on the anode side of the organic LED on top of the culture coverslip immersed in either ambient air or cell medium for various pulse lengths (Figure 3 and Figure S3). We measured the surface temperature for stimuli ranging from 50 to $3,000 \mathrm{~ms}$, mimicking the light stimulation performed with neurons expressing either SSFO or ChrimsonR (Figures 3A,B). The steady-state temperature rise vs. time for three increasing pulse durations (50,500, and 3,000 ms) show insight on the maximum average power that can be safely delivered using this platform for a given DC and $10 \mathrm{kHz}$ OLED operation. As expected, the steadystate temperature increases as a function of the pulse duration for both continuous DC and $10 \mathrm{kHz}$ operation modes. With an average power of $\sim 150 \mu \mathrm{W} / \mathrm{mm}^{2}$ for orange OLEDs and $\sim 100$ $\mu \mathrm{W} / \mathrm{mm}^{2}$ for blue OLEDs, they can safely operate with the $\mathrm{KHz}$ mode with temperature increases $<1^{\circ} \mathrm{C}$ (measured at the end of the pulse; $<2{ }^{\circ} \mathrm{C}$ measured at $7 \mathrm{~s}$ ) for the maximum stimulation period of 3,000 ms while, for the very short pulse period of $50 \mathrm{~ms}$, the temperature increase is only $<0.1^{\circ} \mathrm{C}$ both with $\mathrm{DC}$ and $\mathrm{KHz}$ modes (Figure 3 and Figure S3).

For both DC and pulsed operation in cell medium, the temperature change was strongly dependent on the operating bias. For a DC bias of $7 \mathrm{~V}$, the increase in surface temperature after $7 \mathrm{~s}$ (with OLEDs operated for the first $3 \mathrm{~s}$ ) was a few tenths of a degree for both the blue and orange devices, while it was $\approx 2^{\circ} \mathrm{C}$ for the blue device and $\approx 3^{\circ} \mathrm{C}$ for the orange device for a DC bias of $30 \mathrm{~V}$ (Figures S3a,b,d,e for the blue and orange OLEDs, respectively). Under $10 \mathrm{kHz}$ square-wave operation, the temperature changes were approximately half the size of the DC case (e.g., $\approx 1-1.5^{\circ} \mathrm{C}$ at $30 \mathrm{~V}$ ), consistent with the $50 \%$ duty cycle of the square-wave drive scheme. As expected, the temperature increased with the OLED operating time, with broadly similar temperature rises observed when the devices were in contact with cell culture medium or with air (Figures S3b,e). Notably, these temperature changes did not elicit any depolarization in control neurons not expressing opsins (see below).

The analysis of the external quantum efficiency (EQE) for the blue and orange OLEDs for increasing pulse durations (50, 500, and 3,000 ms) revealed that EQE was relatively similar between continuous and pulsed $(10 \mathrm{KHz})$ operation at the shortest pulse duration $(50 \mathrm{~ms})$. However, at longer times, EQE sharply dropped with DC operation, while it remained relatively constant for pulsed operation (Figures S3c,f and Table 1).

\section{Depolarization of SSFO-Expressing Cortical Neurons by Blue OLEDs}

The coupling of the blue OLED emission to opsin-expressing neurons was investigated using a low-density culture of cortical

TABLE 1 | Summary of EQEs and temperature rise at different pulse durations for DC and $10 \mathrm{kHz}$ operation modes.

\begin{tabular}{|c|c|c|c|c|c|c|c|}
\hline \multicolumn{2}{|c|}{ Operation mode } & \multicolumn{3}{|c|}{ Blue OLED } & \multicolumn{3}{|c|}{ Orange OLED } \\
\hline Pulse period & $\begin{array}{l}\text { Pulse duration } \\
\text { (ms) }\end{array}$ & $\begin{array}{l}\text { Optical power } \\
\left(\mu W / \mathrm{mm}^{2}\right)^{\mathrm{a}}\end{array}$ & $\begin{array}{l}\text { Temperature rise } \\
\left({ }^{\circ} \mathrm{C}\right)^{\mathrm{b}}\end{array}$ & EQE (\%) & $\begin{array}{l}\text { Optical power } \\
\left(\mu \mathrm{W} / \mathrm{mm}^{2}\right)^{\mathrm{a}}\end{array}$ & $\begin{array}{l}\text { Temperature rise } \\
\left({ }^{\circ} \mathrm{C}\right)^{\mathrm{b}}\end{array}$ & EQE (\%) \\
\hline DC & 50 & 96 & 0.02 & 0.29 & 145 & 0.05 & 0.38 \\
\hline $10 \mathrm{kHz}$ & 50 & 99 & 0.006 & 0.34 & 150 & 0.03 & 0.46 \\
\hline DC & 500 & 41 & 0.27 & 0.12 & 74 & 0.53 & 0.19 \\
\hline $10 \mathrm{kHz}$ & 500 & 89 & 0.14 & 0.30 & 143 & 0.25 & 0.43 \\
\hline DC & 3,000 & 6.5 & 0.74 & 0.02 & 61 & 1.13 & 0.16 \\
\hline $10 \mathrm{kHz}$ & 3,000 & 88 & 0.40 & 0.29 & 138 & 0.69 & 0.42 \\
\hline
\end{tabular}

${ }^{a}$ Average optical power during pulse duration.

${ }^{b}$ Temperature rise measured at 3,000 ms. 

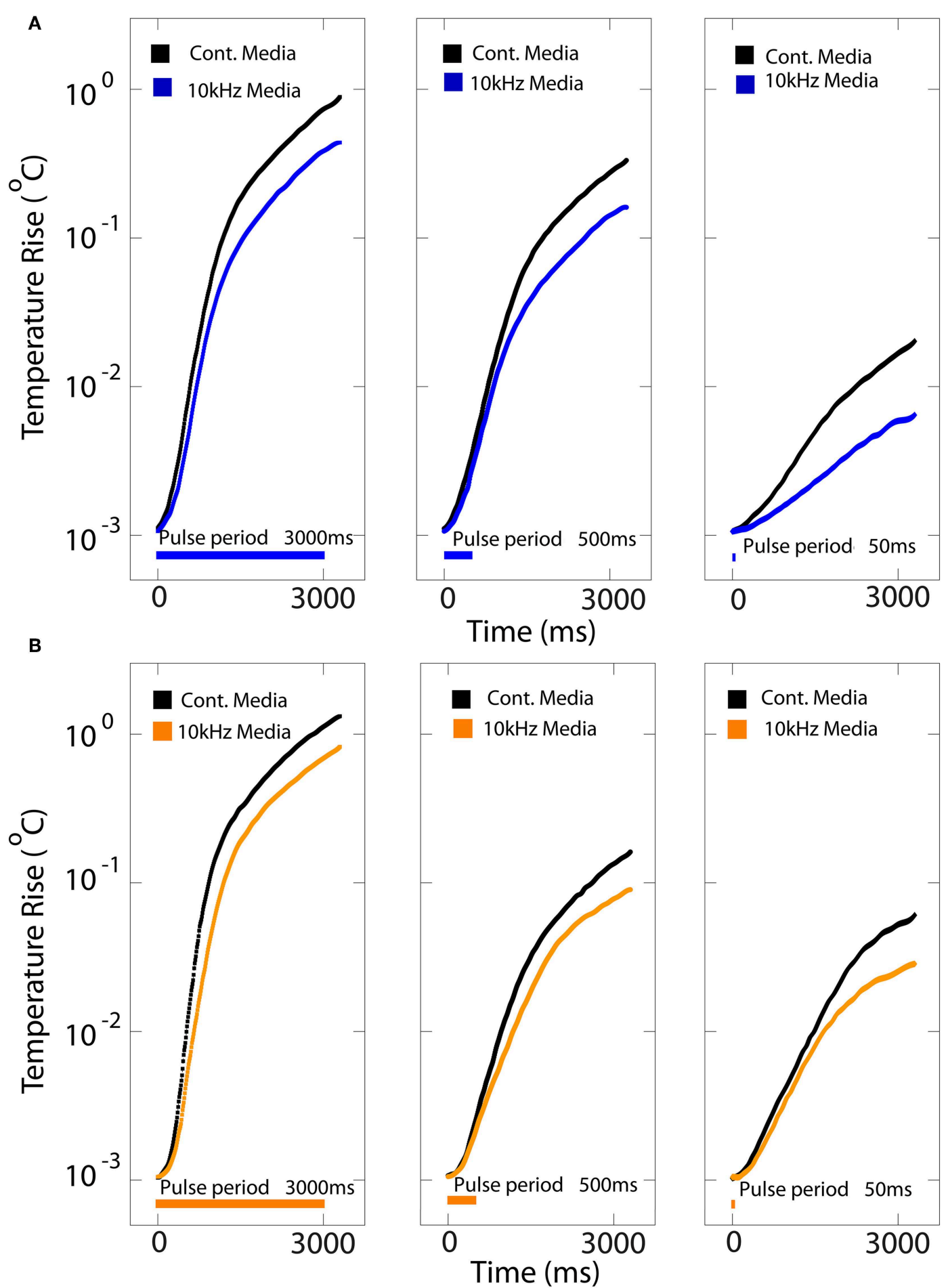

FIGURE 3 | Heating profile of blue and orange OLEDs. The steady-state temperature rise at the surface of a coverslip positioned onto the blue (A) and orange (B) OLEDs immersed in cell-culture medium vs. time for either DC (cont.; black traces) and $10 \mathrm{kHz}$ square-wave (colored traces) stimulation at $30 \mathrm{~V}$ optical power corresponding to for blue and for orange OLEDs for pulse durations of 50, 500, and 3,000 ms.

neurons prepared from E18 mouse embryos. Primary neurons were transfected at 6-8 days in vitro (DIV) with the bi-stable opsin SSFO (Yizhar et al., 2011; Figure 4A); measurements were carried out $24 \mathrm{~h}$ after transfection. SSFO has one of the lowest recorded Effective Power Densities for 50\% activation $\left(\mathrm{EPD}_{50}\right.$; Mattis et al., 2011), lying between 0.1 and $10 \mu \mathrm{W} / \mathrm{mm}^{2}$, 


\section{A}

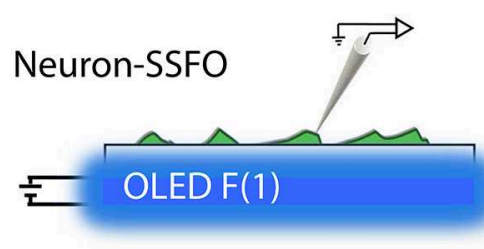

B
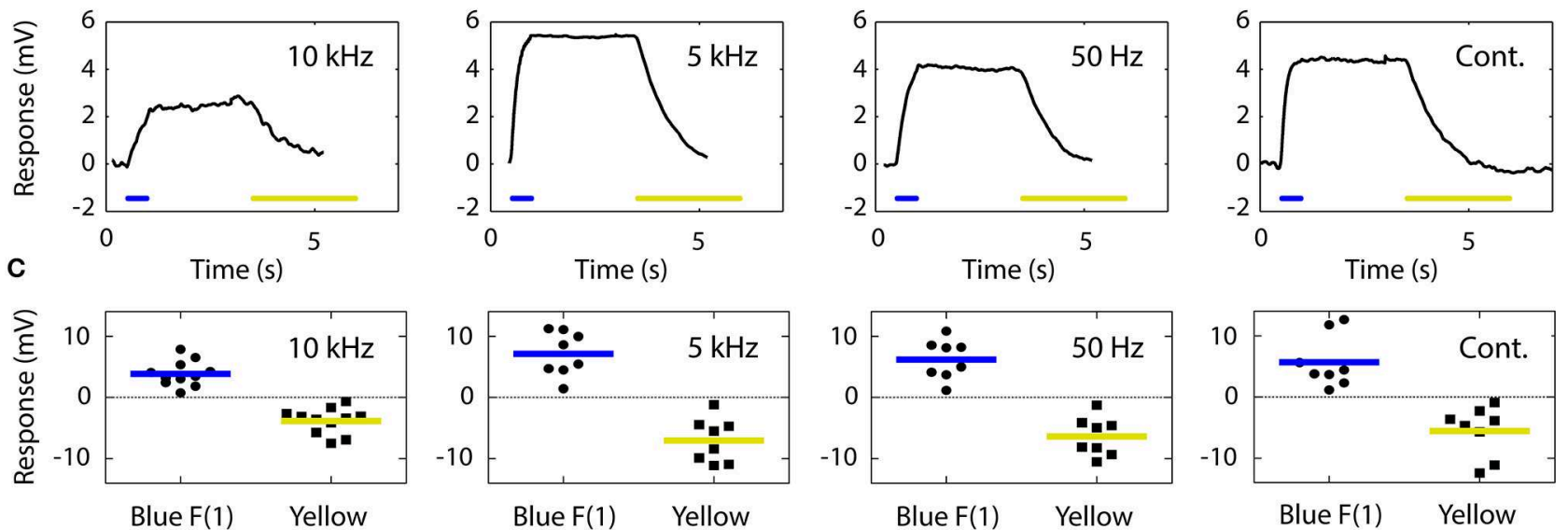

D

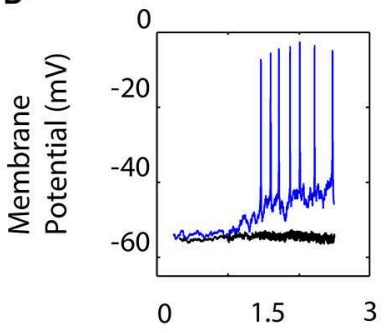

Time (s)
E

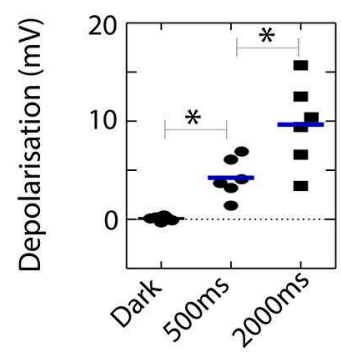

Time Illumination
F

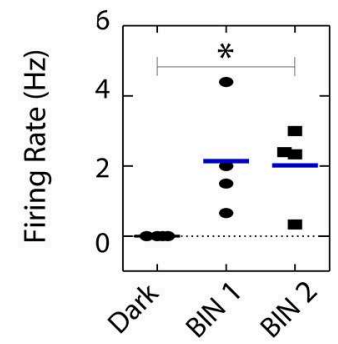

G

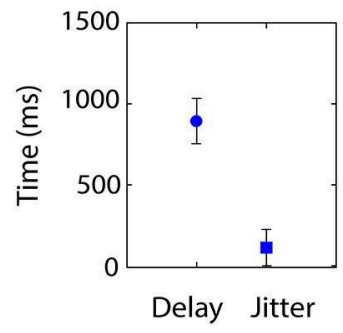

Bin Analyzed

FIGURE 4 | Depolarization of SSFO-expressing neurons by blue OLED illumination at various pulse rates. (A) Neurons were transfected with SSFO, stimulated by a blue OLED, and recorded in current-clamp configuration. (B) Representative responses from individual neurons. At all tested frequencies, 500 ms of blue light stimulation was sufficient to depolarize the neurons, leading to sustained activation that extended beyond the pulse stimulus; activation could be fully reversed by yellow light stimulation. (C) Individual neurons (symbols) and mean (colored lines) amplitudes of blue light-induced depolarization in response to 500 ms stimuli and yellow light-induced hyperpolarization in SSFO-expressing neurons $(n=8-11)$. (D) Sample recording from a mature neuron transfected with SSFO (blue trace) and illuminated for $2 \mathrm{~s}$ that successfully reached the firing threshold. The black trace represents the response of a control mock-transfected neuron. (E) Effects of stimulus length on the extent of depolarization, excluding action potentials, of SSFO-expressing neurons in response to $2 \mathrm{~s}$ stimuli (symbols and lines represent individual neurons and the mean, respectively). Depolarization increased significantly with the time of illumination $(n=6)$. (F) The firing rate of SSFO-expressing neurons increased significantly during prolonged illumination. The individual values (symbols) and mean (line) firing are shown for $1 \mathrm{~s}$ bins before (dark) and during the blue light stimulus $(n=4)$. (G) Quantification of the delay and jitter of action potential firing based on the first spike per stimulus calculated across all recorded neurons.

significantly lower than the measured output of our blue OLEDs in square-wave operation. The low activation power is due to the bi-stable nature of the SSFO opsin channel, which remains in an open conductive state after illumination with blue light, closing with a long relaxation time of $29 \mathrm{~min}$ (Yizhar et al., 2011). This means sequential pulses of light have a cumulative effect on the open channel populations, bypassing the current decay due to the rapid channel inactivation typically experienced by other opsins. Channel closure can be hastened by a secondary pulse of yellow light, causing the membrane potential to return to its resting value (Yizhar et al., 2011). Cell membrane depolarization under blue-light illumination followed by repolarization under yellowlight illumination consequently provides a clear indication that the initial depolarization is attributable to SSFO.

Firstly, we compared the effects of $500 \mathrm{~ms}$ stimuli, driven by a DC bias and $10 \mathrm{kHz}, 5 \mathrm{kHz}$, and $50 \mathrm{~Hz}$ square waves. SSFOtransfected neurons plated on glass coverslips were positioned at the surface of the OLED's anode-side surface $\left(75 \mathrm{~mm}^{2}\right.$ area), with the OLED surface and coverslip bathed in extracellular solution for patch-clamp recordings (Figure 4A; see also Figure 1C). Changes in membrane potential induced by the various light stimulation protocols were recorded in current-clamp mode, 
with each stimulus being repeated five times per recorded cell. The representative traces reported in Figure 4B show that in all cases the emission from the blue OLED successfully activated the SSFO channels and had a depolarizing effect on the recorded neurons. At all tested stimulation frequencies, the depolarization elicited by the blue OLED extended well beyond the $500 \mathrm{~ms}$ duration of the blue light illumination window, and was readily reversed when the neuron was exposed to yellow light (derived from an external yellow inorganic LED focused through the microscope objective), confirming that the voltage responses recorded in the neurons were indeed driven by SSFO-generated currents (Yizhar et al., 2011) (Figure 4B). The mean $( \pm$ sem $)$ response amplitudes measured in the analyzed neurons did not vary significantly across the tested sub-pulse rates (depolarization: $3.9 \pm 0.6 \mathrm{mV}, n=11 ; 7.2 \pm 1.3 \mathrm{mV}, n=8$; $6.2 \pm 1.1 \mathrm{mV}, n=8$; and $5.7 \pm 1.5 \mathrm{mV}, n=8$; for $10 \mathrm{kHz}, 5 \mathrm{kHz}$, $50 \mathrm{~Hz}$, and continuous operation, respectively one-way ANOVA; $p>0.05$ ) (Figure 4C).

We next used a prolonged 2-s stimulation time to drive the SSFO-expressing neurons to the firing threshold. A drive frequency of $10 \mathrm{kHz}$ was employed to ensure sustained device performance and a minimal temperature increase (see Figures 2, 3). Most of the recorded SSFO-expressing neurons $(n=4$ out of 6 neurons) fired multiple action potentials during the period of light emission (Figures 4D-F). A significant increase of firing rate was measured during the illumination period (Kruskal-Wallis/Dunn's tests, $p<0.05$; vs. baseline, $p<0.05$ ). The first spike elicited per stimulus occurred with a delay of $893.7 \pm 139.9 \mathrm{~ms}$ relative to the light stimulus onset and a jitter of $139.9 \pm 112.4 \mathrm{~ms}$ (calculated as the standard deviation of the latency) (Figure 4G). The remaining cells $(n=2$ out of 6) exhibited a substantial depolarization $(6.84 \pm 2.12 \mathrm{mV}$ over the $2 \mathrm{~s}$ illumination period), but did not reach the firing threshold. Mock-transfected neurons, subjected to the same light stimulation conditions, showed no depolarization $(-0.57 \pm$ $1.08 \mathrm{mV}$ ) and no firing during/after the light stimulus.

\section{Millisecond-Scale Control of Spike Firing by Orange OLEDs in ChrimsonR-Expressing Neurons}

The multi-second stimulation procedure used to activate SSFO by the blue OLEDs is suitable for slow tasks like photomediated insulin release or photo-regulation of gene expression, where control on the second-to-minute timescale is typically sufficient. However, many optogenetic applications require opsin currents to be switched on and off with sub-second temporal precision. In addition, it is often preferable to use red light for opsin stimulation since red light penetrates more deeply into tissue and results in lower phototoxicity. We therefore assessed the feasibility of coupling our orange OLEDs to the rapidly inactivating, red shifted opsin ChrimsonR (Chaffiol et al., 2017). To gain high and widespread opsin expression levels in the primary neuronal networks, ChrimsonR was packaged in a lentiviral vector with a high efficiency of neuron transduction (Figure 5A).
ChrimsonR-expressing neurons displayed both sub-threshold depolarizations and light-mediated action potentials in response to stimulation by the orange OLED $(50 \mathrm{~ms}, 10 \mathrm{kHz}, 30 \mathrm{~V})$, while non-transduced neurons were unresponsive to the same light stimuli (Figure 5B and Figure S3a). ChrimsonR-expressing neurons displayed sub-threshold activations of $5.89 \pm 1.52 \mathrm{mV}$ (mean \pm sem; $n=10$ ) during the short $50 \mathrm{~ms}$ stimuli (Figure 5B). These sub-threshold depolarizations were reliably elicited across multiple OLED light stimulations (Figure S3b). In contrast to the SSFO-expressing neurons excited by our blue OLEDs, prolonged illumination $(2 \mathrm{~s}, 10 \mathrm{kHz}, 30 \mathrm{~V})$ of ChrimsonRexpressing neurons by the orange OLEDs led to a mean ( \pm sem) depolarization of $4.3 \pm 1.5 \mathrm{mV}(n=3)$ during the first $500 \mathrm{~ms}$ of illumination that did not further increase during the remainder of the light pulse $(4.01 \pm 1.6 \mathrm{mV}$ at $2,000 \mathrm{~ms}$; see sample trace in Figure 5C).

The largest subset ( $n=13$ out of 26 neurons) of ChrimsonRexpressing neurons showed a reproducible firing pattern in response to light, successfully reaching the firing threshold within the $50 \mathrm{~ms}$ stimulus time (Figure 5D). To check the temporal precision of neuronal activation by the OLED-opsin system, we delivered consecutive light stimuli $(10 \mathrm{kHz}, 50 \mathrm{~ms})$ at various modulation frequencies $(0.5-6.4 \mathrm{~Hz})$. As highlighted by the representative traces of Figure $5 \mathrm{E}$, action potential firing faithfully followed the light cues. The ChrimsonR/orange-OLED system achieved a $100 \%$ firing probability at $0.13 \mathrm{~Hz}(n=6), 94 \%$ at $0.5 \mathrm{~Hz}(n=10), 92.5 \%$ at $1 \mathrm{~Hz}(n=8), 80 \%$ at $3.3 \mathrm{~Hz}(n=$ $7)$, and $70 \%$ at $6.4 \mathrm{~Hz}(n=4)$ showing that, except for occasional failures, neuronal firing patterns were tightly locked to the optical output of the OLED (Figure 5F).

To test the reliability of the system, the neurons were exposed to a long series of repeated stimulations (25-100 stimuli), while holding them under patch-clamp conditions. The experimental protocol employed trains of stimuli composed of five bursts $(0.1 \mathrm{~Hz}$ bursting rate) containing five $50 \mathrm{~ms}$ stimuli administered at $1 \mathrm{~Hz}(10 \mathrm{kHz}$ square wave). The trains were administered up to four times per cell (Figure 5G). The mean firing probability per cell was quantified for each train ( 5 packets of 5 stimuli), revealing a reliable elicitation of action potentials (mean quartile probability values: Q1 $=90.5 \%, n=7 ; \mathrm{Q} 2=93 \% n=5$; $\mathrm{Q} 2=89 \%, n=4$; Q4 = 93\%, $n=4$ ) (Figure $5 \mathrm{H}$ ). A similar performance was obtained using 2-s interstimulus intervals (Q1 $=94 \%, n=4 ; \mathrm{Q} 2=91 \% n=3 ; \mathrm{Q} 2=79 \%, n=3$; $4=76 \%$, $n=3)$. The peristimulus time histogram (PSTH) calculated from the cumulative action potential distribution per cell (Figure 5I) revealed that the increased firing probability closely followed the onset of the light stimulus. Taking into account all firing neurons tested with the orange OLEDs, the mean $( \pm$ sem) time between light onset and the first action potential per stimulus was determined to be $19.2 \pm 3.3 \mathrm{~ms}$, with a jitter of $6.2 \pm 1.1 \mathrm{~ms}$ (Figure 5J). In contrast to ChrimsonR-expressing neurons, nontransduced cells present in the same neural networks were virtually unresponsive to light (mean depolarization in light $=0.09 \pm 0.32 \mathrm{mV}, n=6$ ), except for rare and delayed responses likely mediated by synaptic network connectivity to opsin-expressing neurons (Figures $\mathbf{S 3 c}, \mathbf{d}$ ). In summary, the responses documented here highlight the ability of the orange 


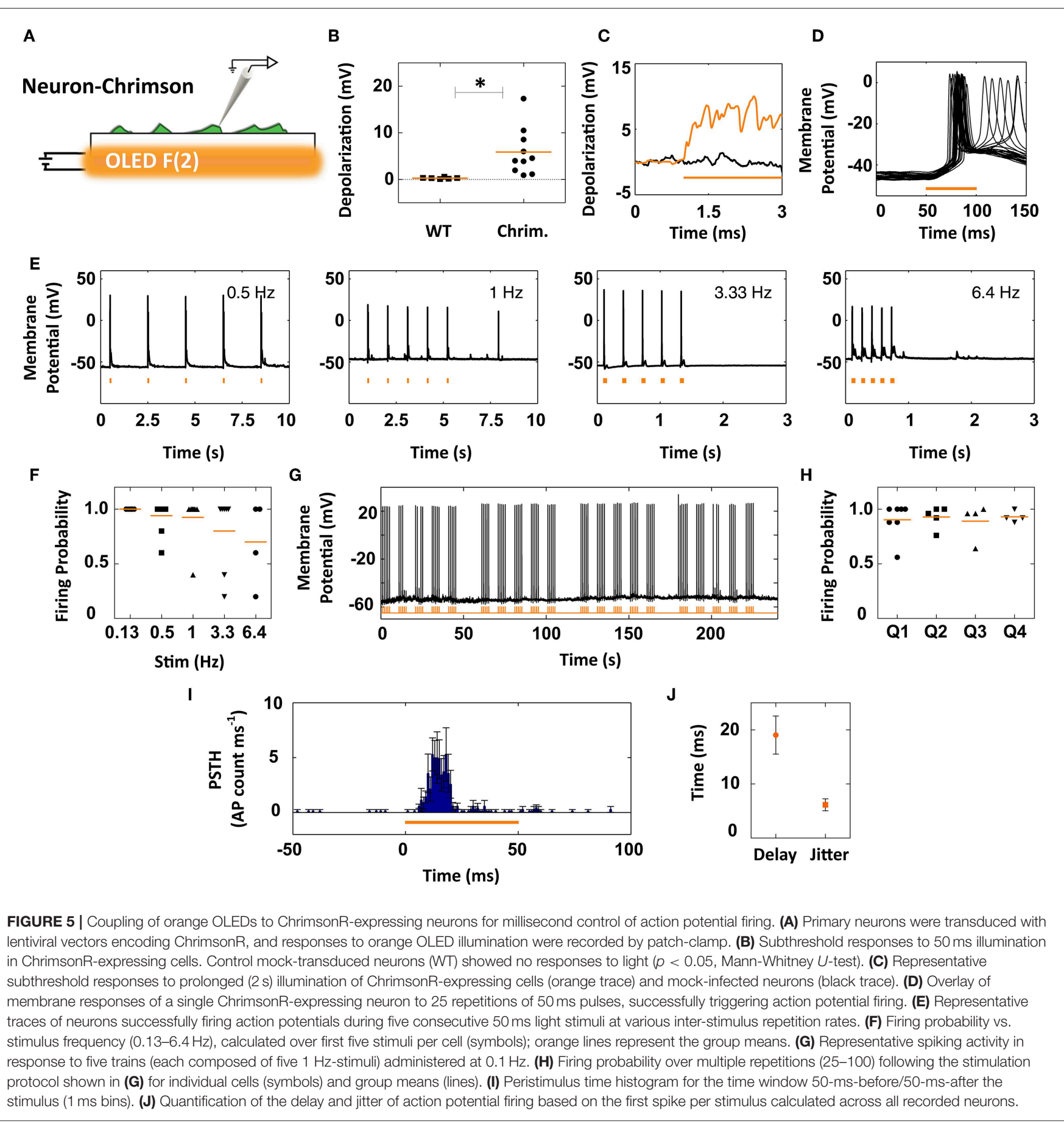

OLED/ChrimsonR couple to photo-stimulate neurons, showing that light-emitting diodes based on organic conjugated polymers can straightforwardly be used to elicit neuronal activation.

\section{DISCUSSION}

The above results indicate the suitability of OLEDs for the optogenetic activation of primary neurons. Devices based on PVK/PFO and SO-PPV were fabricated and characterized and their performance under continuous DC bias and pulse mode operation was compared. While under DC operation, the devices degraded rapidly within a few hundred milliseconds (below the optical powers required for optogenetic stimulation), using square-wave frequencies of $5 \mathrm{kHz}$ and above, they exhibited stable and high brightness output of $>100 \mu \mathrm{W} / \mathrm{mm}^{2}$ when operated at $30 \mathrm{~V}$. For the first time $\mathrm{kHz}$ modulation has been applied to OLEDs for optogenetics applications. Two OLED-Opsin pairs were tested for time-locked neuronal activation with 
multi-kHz pulsing mode. Blue OLEDs successfully stimulated neurons expressing SSFO by inducing a sustained depolarization that persisted beyond the illumination period, and was readily switched off when the neuron was exposed to yellow light, as expected for SSFO-generated currents (Yizhar et al., 2011). Orange OLEDs effectively stimulated neurons expressing the fast inactivating ChrimsonR opsin by eliciting firing patterns were tightly time-locked to the OLED emission that are suitable for most optogenetic applications where opsin currents must be switched on/off on a sub-second timescale.

Previous results using non-solution processed OLEDs, characterized by complex structures and small molecule-type of devices were only able to activate inward currents in opsinexpressing cell lines or induce behavioral changes in opsinexpressing algae or Drosophila larvae on a second scale (Steude et al., 2015, 2016; Morton et al., 2016). Instead, our polymeric light emitting devices made of solution-processable materials were capable to achieve not only of fluctuations in the membrane potential, but a real millisecond-scale control of neuronal firing that is essential for any neuroscience application.

Inorganic LED solutions offer high optical power. However, they lack precise matching with with the opsin activation spectra. Inorganic LEDs are a chip point source (small pixel area) with high power density produced. The generated heat, due to the high power, has very little surface area to dissipate. Inorganic LEDs, which produce a large amount of heat during operation, require additional heat sinking for keeping low temperature of devices. One of the attractive features of polymeric OLEDs is the large-area of homogeneous light emission at relatively low temperature. In addition, OLEDs have the advantage to be used for in vitro electrophysiology coupled to optogenetics. The OLED-Optogenetic platform proposed here can be used to test OLED prototypes before in vivo use on flexible substrates and toward next generation of organic LEDs for optogenetics. The limitations of OLED remain with the relatively short lifetime due to air/aqueous instability of the electron injection layer, which is still a challenge for in vivo use with flexible substrates. Our devices could still operate over $30 \mathrm{~min}$, although some degradation of the optical power occurs overtime, suggesting that the device structure should be optimized for allowing lifetimes needed long-term in vivo applications. However, there is a high demand to incorporate such OLEDs into implantable devices using flexible substrates such as Silicon, Parylene C, Polymide, or SU8 with appropriate oxygen barrier materials. Although fully polymeric OLEDs are less efficient that inorganic LEDs, the device performance could be further enhanced when using highly sensitive opsins that require lower density operation. This will pave the way for a near future of fully biocompatible, stable and highly performance of fully Polymeric OLEDs.

\section{CONCLUSIONS}

Currently encapsulated in glass, the described OLEDs are can be employed as smart culture substrates for in vitro optogenetics experiments. Indeed, a series of OLEDs capable of meeting the lowest activation requirements of the most commonly used optogenetic actuators is shown in Figure S4. Future work will focus on the development of more efficient and stable devices that allow using lower drive voltages and extended operation times, as well as on the development of thin-film embeddable devices for in vivo optogenetic applications. Ultimately, the promise of OLED technology is a system that is easily implementable, implantable, and devoid of external optics. The rapid pace of progress in OLED and optogenetic technologies will ensure that OLEDs continue to find numerous applications in bioelectronics.

\section{DATA AVAILABILITY STATEMENT}

The authors declare that all data that support the figures within this paper and other findings of this study are hosted at the Istituto Italiano di Tecnologia and can be accessed by contacting the corresponding author.

\section{ETHICS STATEMENT}

The animal study was reviewed and approved by Italian Ministry of Health.

\section{AUTHOR CONTRIBUTIONS}

BM designed, fabricated and characterized devices, and prepared hardware communication protocols for OLED integration with patch-clamp set-up. PF prepared neuronal cultures, produced viruses, carried out electrophysiological recordings, and contributed to device design. JM and FB planned experiments and contributed critically to data analysis and interpretation. All authors contributed to the writing of the manuscript.

\section{FUNDING}

This work was supported by EU through project FP7-PEOPLE212-ITN 316832-OLIMPIA (to FB and JM) and by Fondazione Cariplo (project 2018-0505 to FB).

\section{ACKNOWLEDGMENTS}

The authors thank Dr. Luigi Naldini (Tiget, Milano, Italy) for lentiviral constructs and protocols; Drs. K. Deisseroth (Stanford University, Stanford, CA) and Edward Boyden (MIT, Cambridge, MA) for kindly providing the opsin plasmids; Arta Mehilli and Diego Moruzzo (Istituto Italiano di Tecnologia, Genova, Italy) for assistance with the preparation of primary cultures and viral constructs.

\section{SUPPLEMENTARY MATERIAL}

The Supplementary Material for this article can be found online at: https://www.frontiersin.org/articles/10.3389/fbioe. 2019.00278/full\#supplementary-material 


\section{REFERENCES}

Beiert, T., Bruegmann, T., and Sasse, P. (2014). Optogenetic activation of Gq signalling modulates pacemaker activity of cardiomyocytes. Cardiovasc. Res. 102, 507-516. doi: $10.1093 / \mathrm{cvr} / \mathrm{cvu} 046$

Blain Christen, J., Kullman, D., Muthuswamy, J., O'Brien, B., Sridharan, A., Lee, Y., et al. (2016). Optogenetic neurostimulation of auricular vagus using flexible OLED display technology to treat chronic inflammatory disease and mental health disorders. Electron. Lett. 52, 900-902. doi: 10.1049/el.2015.3450

Bruegmann, T., Van Bremen, T., Vogt, C., Send, T., Fleischmann, B., and Sasse, P. (2015). Optogenetic control of contractile function in skeletal muscle. Nat. Commun. 6:7153. doi: 10.1038/ncomms8153

Cehajic-Kapetanovic, J., Eleftheriou, C., Allen, A., Milosavljevic, N., Pienaar, A., Bedford, R., et al. (2015). Restoration of vision with ectopic expression of human rod opsin. Curr. Biol. 25, 2111-2122. doi: 10.1016/j.cub.2015.07.029

Chaffiol, A., Caplette, R., Jaillard, C., Brazhnikova, E., Desrosiers, M., Dubus, E., et al. (2017). A new promoter allows optogenetic vision restoration with enhanced sensitivity in macaque retina. Mol. Ther. 25, 2546-2560. doi: 10.1016/j.ymthe.2017.07.011

Chuong, A. S., Miri, M. L., Busskamp, V., Matthews, G. A., Acker, L. C., Sørensen, A. T., et al. (2014). Noninvasive optical inhibition with a red-shifted microbial rhodopsin. Nat. Neurosci. 17, 1123-1129. doi: 10.1038/nn.3752

Dal Maschio, M., Difato, F., Beltramo, R., Blau, A., Benfenati, F., and Fellin, T. (2010). Simultaneous two-photon imaging and photo-stimulation with structured light illumination. Opt. Express 18, 18720-18731. doi: 10.1364/OE.18.018720

Dawydow, A., Gueta, R., Ljaschenko, D., Ullrich, S., Hermann, M., Ehmann, N., et al. (2014). Channelrhodopsin-2-XXL, a powerful optogenetic tool for low-light applications. Proc. Natl. Acad. Sci. U.S.A. 111, 13972-13977. doi: 10.1073/pnas.1408269111

Falco, A., Matarese, B., Feyen, P., Benfenati, F., Lugli, P., and de Mello, J. C. (2016). Investigation of the stability and biocompatibility of commonly used electrode materials in organic neuro-optoelectronics. IEEE Trans. Nanotech. 15, 746-753. doi: 10.1109/TNANO.2016.2536946

Fan, B., and Li, W. (2015). Miniaturized optogenetic neural implants: a review. Lab. Chip 15, 3838-3855. doi: 10.1039/C5LC00588D

Gaub, B., Berry, M., Holt, A., Isacoff, E., and Flannery, J. (2015). Optogenetic vision restoration using rhodopsin for enhanced sensitivity. Mol. Ther. 23, 1562-1571. doi: 10.1038/mt.2015.121

Ghezzi, D., Antognazza, M. R., Dal Maschio, M., Lanzarini, E., Benfenati, F., and Lanzani, G. (2011). A hybrid bioorganic interface for neuronal photoactivation. Nat. Commun. 2:166. doi: 10.1038/ncomms1164

Ghezzi, D., Antognazza, M. R., Maccarone, R., Bellani, S., Lanzarini, E., Martino, N., et al. (2013). A polymer optoelectronic interface restores light sensitivity in blind rat retinas. Nat. Phot. 7, 400-406. doi: 10.1038/nphoton.2013.34

Gossler, C., Bierbrauer, C., Moser, R., Kunzer, M., Holc, K., Pletschen, W., et al. (2014). T. GaN-based micro-LED arrays on flexible substrates for optical cochlear implants. J. Phys. D Appl. Phys. 47:205401. doi: 10.1088/0022-3727/47/20/205401

Govorunova, E. G., Sineshchekov, O. A., Janz, R., Liu, X., and Spudich, J. L. (2015). Natural light-gated anion channels: a family of microbial rhodopsins for advanced optogenetics. Science 349, 647-650. doi: 10.1126/science.aaa7484

Grossman, N., Poher, V., Grubb, M. S., Kennedy, G. T., Nikolic, K., McGovern, B., et al. (2010). Multi-site optical excitation using ChR2 and micro-LED array. J. Neural Eng. 7:016004. doi: 10.1088/1741-2560/7/1/016004

Grusch, M., Schelch, K., Riedler, R., Reichhart, E., Differ, C., Berger, W., et al. (2014). Spatio-temporally precise activation of engineered receptor tyrosine kinases by light. $E M B O$ J. 33, 1713-1726. doi: 10.15252/embj.2013 87695

Kaufmann, J. C. D., Benjamin, S., Krause, B. S., Grimm, C., Ritter, E., Hegemann, P., et al. (2017). Proton transfer reactions in the red light-activatable channelrhodopsin variant ReaChR and their relevance for its function. J. Biol. Chem. 292, 14205-14216. doi: 10.1074/jbc.M117.779629

Klapoetke, N., Murata, Y., Kim, S., Pulver, S., Birdsey-Benson, A., Cho, Y., et al. (2014). Independent optical excitation of distinct neural populations. Nat. Methods 11, 338-346. doi: 10.1038/nmeth.2836

Krames, M. R., Shchekin, O. B., Mueller-Mach, R., Mueller, G. O., Zhou, L., Harbers, G., et al. (2007). Status and future of high-power light-emitting diodes for solid-state lighting. J. Display Tech. 3, 160-175. doi: 10.1109/JDT.2007.895339

Lochner, C. M., Khan, Y., Pierre, A., and Arias, A. C. (2014). Allorganic optoelectronic sensor for pulse oximetry. Nat. Commun. 5:5745. doi: $10.1038 /$ ncomms6745

Maimon, B. E., Zorzos, A. N., Bendell, R., Harding, A., Fahmi, M., Srinivasan, S., et al. (2017). Transdermal optogenetic peripheral nerve stimulation. J. Neural Eng. 14:34002. doi: 10.1088/1741-2552/aa5e20

Matarèse, B. (2017). Organic Light Emitting Diodes (OLEDs) for Neurooptoelectronics (Ph.D. Thesis). 1-215. Available online at: http://hdl.handle.net/ 10044/1/68710.

Matarèse, B. F. E., Feyen, P. L. C., Falco, A., Benfenati, F., Lugli, P., and deMello, J. C. (2018). Use of SU8 as a stable and biocompatible adhesion layer for gold bioelectrodes. Sci. Rep. 8:5560. doi: 10.1038/s41598-018-21755-6

Mattis, J., Tye, K. M., Ferenczi, E. A., Ramakrishnan, C., O’Shea, D. J., Prakash, R., et al. (2011). Principles for applying optogenetic tools derived from direct comparative analysis of microbial opsins. Nat. Meth. 9, 159-172. doi: $10.1038 /$ nmeth. 1808

Mohammad, F., Stewart, J., Ott, S., Chlebikova, K., Chua, J., Koh, T., et al. (2017). Optogenetic inhibition of behavior with anion channelrhodopsins. Nat. Methods 14, 271-274. doi: 10.1038/nmeth.4148

Morton, A., Murawski, C., Pulver, S., and Gather, M. (2016). High-brightness organic light-emitting diodes for optogenetic control of Drosophila locomotor behaviour. Sci. Rep. 6:31117. doi: 10.1038/srep31117

Nakanotani, H., Oyamada, T., Kawamura, Y., Sasabe, H., and Adachi, C. (2005). Injection and transport of high current density over $1000 \mathrm{~A} / \mathrm{cm} 2$ in organic light emitting diodes under pulse excitation. Jpn. J. Appl. Phys. 44, 3659-3662. doi: 10.1143/JJAP.44.3659

Packer, A. M., Peterka, D. S., Hirtz, J. J., Prakash, R., Deisseroth, K., and Yuste, R. (2012). Two-photon optogenetics of dendritic spines and neural circuits. Nat. Meth. 9, 1202-1205. doi: 10.1038/nmeth.2249

Paonessa, F., Criscuolo, S., Sacchetti, S., Amoroso, D., Scarongella, H., Pecoraro Bisogni, F., et al. (2016). Regulation of neural gene transcription by optogenetic inhibition of the RE1-silencing transcription factor. Proc. Natl. Acad. Sci. U.S.A. 113, E91-E100. doi: 10.1073/pnas.1507355112

Park, S.-I., Xiong, Y., Kim, R.-H., Elvikis, P., Meitl, M., Kim, D.-H., et al. (2009). Printed assemblies of inorganic light-emitting diodes for deformable and semitransparent displays. Science 325, 977-981. doi: 10.1126/science.1175690

Park, S.-J., Gazzola, M., Park, K. S., Park, S., Di Santo, V., Blevins, E. L., et al. (2016). Phototactic guidance of a tissue-engineered soft-robotic ray. Science 353, 158-162. doi: 10.1126/science.aaf4292

Paz, J., and Huguenard, J. (2015). Optogenetics and epilepsy: past, present and future. Epilepsy Curr. 15, 34-38. doi: 10.5698/1535-7597-15.1.34

Qazi, R., Kim, C. Y., Byun, S., Jeong, J., and Vittorio, M. D. (2018). Microscale inorganic LED based wireless neural systems for chronic in vivo optogenetics. Front. Neurosci. 12:764. doi: 10.3389/fnins.2018.00764

Raffelberg, S., Wang, L., Gao, S., Losi, A., Gärtner, W., and Nagel, G. (2013). A LOV-domain-mediated blue-light-activated adenylate (adenylyl) cyclase from the cyanobacterium Microcoleus chthonoplastes PCC 7420. Biochem. J. 455, 359-365. doi: 10.1042/BJ20130637

Reinbothe, T. M., Safi, F., Axelsson, A. S., Mollet, I. G., and Rosengren, A. H. (2014). Optogenetic control of insulin secretion in intact pancreatic islets with $\beta$-cell-specific expression of Channelrhodopsin-2. Islets 6:e28095. doi: $10.4161 /$ isl.28095

RST-001 Phase I/II Trial for Retinitis Pigmentosa (2016). ClinicalTrials.gov. Available online at: https://clinicaltrials.gov/ct2/show/ NCT02556736?term= optogeneticsandrank=1.

Sandström, A., Dam, H., Krebs, F., and Edman, L. (2012). Ambient fabrication of flexible and large-area organic light-emitting devices using slot-die coating. Nat. Commun. 3:1002. doi: 10.1038/ncomms2002

Smith, J. T., O’Brien, B., Lee, Y. K., Bawolek, E. J., and Christen, J. B. (2014). Application of flexible OLED display technology for electro-optical stimulation and/or silencing of neural activity. J. Disp. Technol. 10, 514-520. doi: 10.1109/JDT.2014.2308436

Soltan, A., Zhao, H., Chaudet, L., Neil, M., Maaskant, P., and Degenaar, P. (2014). "An 8100 pixel optoelectronic array for optogenetic retinal prosthesis," in 2014 IEEE Biomedical Circuits and Systems Conference (BioCAS) Proceedings (Lausanne), 352-355. doi: 10.1109/BioCAS.2014.6981735 
Someya, T., Bao, Z., and Malliaras, G. (2016). The rise of plastic bioelectronics. Nature 540, 379-385. doi: 10.1038/nature21004

Steude, A., Jahnel, M., Thomschke, M., Schober, M., and Gather, M. C. (2015). Controlling the behavior of single live cells with high density arrays of microscopic OLEDs. Adv. Mater. 27, 7657-7661. doi: 10.1002/adma.201503253

Steude, A., Witts, E. C., Miles, G. B., and Gather, M. C. (2016). Arrays of microscopic organic LEDs for high-resolution optogenetics. Sci. Adv. 2:e1600061. doi: 10.1126/sciadv.1600061

Stierl, M., Stumpf, P., Udwari, D., Gueta, R., Hagedorn, R., Losi, A., et al. (2011). Light modulation of cellular cAMP by a small bacterial photoactivated adenylyl cyclase, bPAC, of the soil bacterium Beggiatoa. J. Biol. Chem. 286, 1181-1188. doi: 10.1074/jbc.M110.185496

Toettcher, J. E., Voigt, C. A., Weiner, O. D., and Lim, W. A. (2011). The promise of optogenetics in cell biology: interrogating molecular circuits in space and time. Nat. Meth. 8, 35-38. doi: 10.1038/nmeth.f.326

Toffanin, S., Benfenati, V., Pistone, A., Bonetti, S., Koopman, W., Posati, T., et al. (2013). N-type perylene-based organic semiconductors for functional neural interfacing. J. Mater. Chem. B 1, 3850-3859. doi: 10.1039/c3tb20555j

Tsintzos, S. I., Pelekanos, N. T., Konstantinidis, G., Hatzopoulos, Z., and Savvidis, P. G. (2008). A GaAs polariton light-emitting diode operating near room temperature. Nature 453, 372-375. doi: 10.1038/nature06979

Welkenhuysen, M., Hoffman, L., Luo, Z., De Proft, A., Van Den Haute, C., Baekelandt, V., et al. (2016). An integrated multi-electrode-optrode array for in vitro optogenetics. Sci. Rep. 6:20353. doi: 10.1038/srep20353

White, M., Kaltenbrunner, M., Głowacki, E., Gutnichenko, K., Kettlgruber, G., Graz, I., et al. (2013). Ultrathin, highly flexible and stretchable PLEDs. Nat. Phot. 7, 811-816. doi: 10.1038/nphoton.2013.188

Wietek, J., Beltramo, R., Scanziani, M., Hegemann, P., Oertner, T. G., and Wiegert, J. S. (2015). An improved chloride-conducting channelrhodopsin for light-induced inhibition of neuronal activity in vivo. Sci. Rep. 5:14807. doi: $10.1038 /$ srep 14807

Williams, D. C., Bejjani, R. E., Ramirez, P. M., Coakley, S., Kim, S. A., Lee, H., et al. (2013). Rapid and permanent neuronal inactivation in vivo via subcellular generation of reactive oxygen with the use of KillerRed. Cell Rep. 5, 553-563. doi: 10.1016/j.celrep.2013.09.023
Yang, H., Lightner, C. R., and Dong, L. (2012). Light-emitting coaxial nanofibers. ACS Nano 6, 622-628. doi: 10.1021/nn204055t

Yin, D., Feng, J., Ma, R., Liu, Y., Zhang, Y., Zhang, X., et al. (2016). Efficient and mechanically robust stretchable organic light-emitting devices by a laser-programmable buckling process. Nat. Commun. 7:11573. doi: $10.1038 /$ ncomms 11573

Yizhar, O., Fenno, L., Prigge, M., Schneider, F., Davidson, T., Ogshea, D., et al. (2011). Neocortical excitation/inhibition balance in information processing and social dysfunction. Nature 477, 171-178. doi: 10.1038/nature 10360

Yokota, T., Zalar, P., Kaltenbrunner, M., Jinno, H., Matsuhisa, N., Kitanosako, H., et al. (2016). Ultraflexible organic photonic skin. Sci. Adv. 2:e1501856. doi: 10.1126/sciadv.1501856

Yu, J., Chen, K., Lucero, R. V., Ambrosi, C. M., and Entcheva, E. (2015). Cardiac optogenetics: enhancement by all-trans-retinal. Sci. Rep. 5:16542. doi: $10.1038 /$ srep 16542

Zhang, Z., Guo, K., Li, Y., Li, X., Guan, G., Li, H., et al. (2015). A colour-tunable, weavable fibre-shaped polymer light-emitting electrochemical cell. Nat. Phot. 9, 233-238. doi: 10.1038/nphoton. 2015.37

Zheng, H., Zheng, Y., Liu, N., Ai, N., Wang, Q., Wu, S., et al. (2013). All-solution processed polymer light-emitting diode displays. Nat. Commun. 4, 1858-1863. doi: $10.1038 /$ ncomms 2971

Conflict of Interest: The authors declare that the research was conducted in the absence of any commercial or financial relationships that could be construed as a potential conflict of interest.

Copyright (C) 2019 Matarèse, Feyen, de Mello and Benfenati. This is an open-access article distributed under the terms of the Creative Commons Attribution License (CC $B Y)$. The use, distribution or reproduction in other forums is permitted, provided the original author(s) and the copyright owner(s) are credited and that the original publication in this journal is cited, in accordance with accepted academic practice. No use, distribution or reproduction is permitted which does not comply with these terms. 